\title{
ORIGINAL
}

\section{INTERRUPCIÓN VOLUNTARIA DEL EMBARAZO EN MUJERES DE UN DISTRITO SANITARIO DE ALMERÍA DURANTE EL PERÍODO 1998-2002}

\author{
Pilar Barroso García (1), Ma Ángeles Lucerna Méndez (2) y Tesifón Parrón Carreño (3) \\ (1) Sección de epidemiología. Distrito Sanitario de Atención Primaria Levante Alto Almanzora. Almería. \\ (2) Servicio de Medicina Preventiva. Hospital la Inmaculada. Huércal-Overa. Almería. \\ (3) Servicio de Salud. Delegación Provincial de Salud. Almería.
}

\section{RESUMEN}

Fundamento: En Andalucía y concretamente en Almería, ha aumentado la población inmigrante. Los distritos sanitarios de atención primaria gestionan las solicitudes de interrupción voluntaria de embarazo. El objetivo de este trabajo es describir la evolución de las solicitudes de interrupción voluntaria de embarazo en mujeres inmigrantes y no inmigrantes en el Distrito Sanitario Levante-Alto Almanzora de Almería.

Métodos: Estudio descriptivo realizado para el período 1998 2002. Material de estudio: solicitudes de interrupción voluntaria de embarazo, datos demográficos del IEA, tarjetas de reconocimiento temporal. Variables estudiadas: Edad, fecha de solicitud, gestación $\geq$ o < a 12 semanas, tipo de población según fuera inmigrante o no. Se calcularon tasas de interrupción voluntaria de embarazo por 1.000 mujeres de 15 a 49 años y el riesgo relativo con intervalos de confianza y significación estadística para estudiar relación entre número de interrupciones y tipo de población.

Resultados: Se estudiaron 224 solicitudes. El mayor número se produjo en el año 2002, tasa de 3,1. El grupo de edad con más solicitudes fue el de 20-24 años (29,5\%). El 95\% de las mujeres presentaba gestación inferior a 12 semanas. El 52,7\% eran mujeres inmigrantes. Con mayor riesgo de solicitar interrupción voluntaria de embarazo en inmigrantes respecto a autóctonas, RR de 66,45 (IC 95\%: 36,11-122,28) en 2001; 292,8 (IC 95\%: 91,12-940,9) en 1999.

Conclusiones: En el período estudiado se observa un aumento de la interrupción voluntaria de embarazo en los servicios públicos, sobre todo en mujeres inmigrantes.

Palabras clave: Solicitantes de aborto. Aborto eugénico. aborto inducido. Servicios de planificación familiar. Anticoncepción.

Correspondencia:

Pilar Barroso García

Levante Alto Almanzor

C/ Silvestre Martínez de Haro, s/n

Huércal-Overa

04600 Almería

Correo electrónico: mariap.barroso.sspa@juntadeandalucia.es
ABSTRACT

Voluntary Interruption of Pregnancy
among Women in an Health District
within the 1998-2002 Period. Almeria,
Spain

Background: The immigrant population has undergone an increase in Andalusia as a whole and in Almeria in particular. The primary healthcare districts manage the requests for voluntary interruption of pregnancy. This study is aimed as describing the trend in requests for voluntary interruption of pregnancy among immigrant and non-immigrant women in the "Levante-Alto Almanzora" Health District of Almeria.

Methods: Descriptive study conducts during the 1998-2002 period. Study material: requests for voluntary interruption of pregnancy, Andalusian Institute of Statistics demographic data, temporary medical care cards. Variables studied: Age, date of request, pregnancy $\geq$, or $<12$ weeks, type of population (immigrant or non-immigrant). Voluntary interruption of pregnancy rates were calculated per 1000 women within the 15-49 age range and the relative risk with confidence intervals and statistical significance for studying the relationship between the number of interruptions and populations type.

Results: A total of 224 requests were studied. The largest number were made in 2002, for a 3.1 rate. The age group making the largest number of requests was the $20-24$ age range $(29.5 \%)$. Ninetyfive percent $(95 \%)$ of the women were less than 12-weeks pregnant. A total of $52.7 \%$ were immigrant women, a greater risk of requesting voluntary interruption of pregnancy having been found among immigrants as compared to native Spanish women, RR of 66.45 (CI 95\%: 36.11-122.28) in 2001; 292.8 (CI 95\%: 91.12-940.9) in 1999.

Conclusions: Voluntary interruption of pregnancy was found to have increased in the public services, particularly among immigrant women, within the period studied.

Key words: Abortion applicant. Abortion, eugenic. Abortion induced. Family Planning Services. Contraception. 


\section{INTRODUCCIÓN}

La planificación familiar está reconocida como una intervención efectiva en Salud Pública ${ }^{1}$. Para reducir el aborto inducido se considera necesario disponer de buenos servicios de planificación familiar en todos los países, así como del desarrollo de programas educativos y escuchar la opinión de las mujeres $^{2-7}$. En la actualidad, la anticoncepción de emergencia podría servir para reducir embarazos no deseados y abortos de ries$\mathrm{go}^{8}$.

Ante circunstancias y riesgos no deseados la mujer recurre a la interrupción voluntaria del embarazo (IVE), variando las solicitudes según las zonas. Así encontramos que en España las tasas de IVE por 1.000 mujeres de entre 15 y 44 años oscilan entre 6 y 7,7 en el período 1998-2001. Por Comunidades Autónomas Andalucía presenta unas tasas algo más bajas que oscilan entre 5,1 y 6,8 para el mismo período, y otras Comunidades como Madrid y Cataluña presentan valores más altos: 10,5 y 10,2 para el año 2001 respectivamente $^{9}$. En otros países encontramos mayor oscilación en los datos. Así en los Países Bajos se registra una tasa de 6,5, y en el Este de Europa, en Rumanía de $78^{3}$. Aunque existe poca bibliografía sobre aborto en mujeres inmigrantes, algunos estudios realizados en distintos países como Suecia, Países Bajos e Inglaterra han encontrado mayor frecuencia de aborto inducido en las mujeres inmigrantes procedentes del Caribe y de la antigua Yugoslavia que en las autóctonas ${ }^{10-12}$.

En España se inició el Registro de IVE en $1986^{13}$ y en la Comunidad Autónoma de Andalucía en 1987. Los centros públicos y privados donde se realizan IVE remiten los cuestionarios a la Consejería de Salud y de ahí se derivan al Ministerio de Sanidad y Consumo ${ }^{9}$. La gestión de las IVE se realiza a través de los distritos sanitarios. En el Distrito Levante-Alto Almanzora de Almería, el médico de familia cumplimenta la solicitud correspondiente y deriva a la mujer al distri- to, dónde se le solicita la cita con la clínica concertada y se le indican medidas de educación sanitaria. En la actualidad hay dos clínicas concertadas, ubicadas en Almería (hasta el año 2001 se derivaban a una de Málaga) y en Sevilla, con las que se gestionan dos de los tres supuestos legales ${ }^{14}$ : peligro para la vida o la salud física o psíquica de la madre, y violación. En el tercero de los supuestos, presunción de graves taras físicas o psíquicas en el feto, el Servicio de Ginecología del Hospital «La Inmaculada» de Huércal-Overa, detecta estas alteraciones realizando la IVE sin derivar a la mujer al distrito sanitario.

Desde el año 1999, en el distrito se ha producido un aumento progresivo de la población inmigrante, que recibe asistencia sanitaria mediante tarjetas de reconocimiento temporal, que tienen validez de un año, hasta que se regulariza su situación ${ }^{15}$. Su número ha aumentado de 48 en el año 1999, a 1973 en el año 2002. La mayoría de los inmigrantes proceden de Sudamérica, sobre todo de Ecuador, y de Europa del Este. En los últimos años se ha detectado que las mujeres inmigrantes, cada vez en mayor número, solicitan IVE, por ello se planteó una investigación con el objetivo de describir la evolución temporal del número de solicitudes de IVE en mujeres inmigrantes y no inmigrantes en el Distrito Sanitario Levante-Alto Almanzora de Almería en el período 19982002.

\section{MATERIAL Y MÉTODOS}

Se trata de un estudio observacional descriptivo de las solicitudes de IVE del Distrito Levante-Alto Almanzora, derivadas a centros concertados en el período 19982002. Dicho distrito es de ámbito rural y está situado en la zona norte de la provincia de Almería. Su población es de 115.007 habitantes según padrón del año 2001. Está dividido en siete Zonas Básicas de Salud (ZBS). 
El material analizado fueron las solicitudes de IVE correspondientes a los dos primeros supuestos legales de todas las ZBS gestionadas por el distrito sanitario. En ellas se recogen datos identificativos como el número de documento nacional de identidad o pasaporte y el de la tarjeta sanitaria, además de otros datos como edad y semana de gestación.

Se estudiaron las siguientes variables: edad; fecha de solicitud; gestación menor, mayor o igual de 12 semanas y si la mujer era o no inmigrante. Como fuentes de información se utilizaron: las solicitudes de IVE derivadas y los datos de Proyección de población por ámbitos subregionales publicados por el Instituto de Estadística de Andalucía (IEA). Para el cálculo de tasas por 1.000 mujeres de 15 a 49 años en población no inmigrante se emplearon los datos del IEA de cada año, para ese grupo de edad. y

Tabla 1

Solicitudes de IVE por años, grupos de edad, semanas de gestación y tipo de población

\begin{tabular}{|c|c|c|c|}
\hline & Número & Porcentaje & $\begin{array}{l}\text { Tasas anuales x } 1000 \text { mujeres de } \\
15-49 \text { años }\end{array}$ \\
\hline \multicolumn{4}{|l|}{ Años } \\
\hline 1998 & 32 & 14,3 & 1,2 \\
\hline 1999 & 17 & 7,6 & 0,6 \\
\hline 2000 & 42 & 18,8 & 1,5 \\
\hline 2001 & 44 & 19,6 & 1,6 \\
\hline 2002 & 89 & 39,7 & 3,1 \\
\hline Total & 224 & 100 & \\
\hline \multicolumn{4}{|l|}{ Edad } \\
\hline $15-19$ & 20 & 8,9 & -- \\
\hline $20-24$ & 66 & 29,5 & -- \\
\hline $25-29$ & 50 & 22,3 & -- \\
\hline $30-34$ & 41 & 18,3 & -- \\
\hline $35-39$ & 31 & 13,8 & -- \\
\hline $40-44$ & 13 & 5,8 & -- \\
\hline $45-49$ & 3 & 1,3 & -- \\
\hline Total & 224 & 100 & \\
\hline Semanas de gestación & & & $\begin{array}{l}\text { Tasas medias x } 1000 \text { mujeres de } \\
15-49 \text { años }(1998-2002)\end{array}$ \\
\hline$<12$ semanas & 211 & 95 & 1,5 \\
\hline$\geq 12$ semanas & 11 & 5 & 0,08 \\
\hline Total & 222 & 100 & \\
\hline \multicolumn{4}{|l|}{ Tipo de población } \\
\hline Inmigrante & 118 & 52,7 & 62,2 \\
\hline No inmigrante & 106 & 47,3 & 0,8 \\
\hline Total & 224 & 100 & \\
\hline
\end{tabular}

En población no inmigrante se han utilizado datos de Proyección de población por ámbitos subregionales (IEA): 27164 en 1998 , 27338 en 1999, 27451 en 2000, 27606 en 2001 y 27697 en 2002; y en las mujeres inmigrantes Tarjetas de Reconocimiento Temporal: 0 en 1998, 20 en 1999, 388 en 2000, 728 en 2001 y 760 en 2002. 
las tarjetas de reconocimiento temporal de asistencia sanitaria realizadas anualmente para las mujeres inmigrantes gracias al Convenio de colaboración en materia de Salud Pública para el colectivo de inmigrantes, firmado en el año 1999 entre la Consejería de Salud y distintas organizaciones ${ }^{15}$, dado que en el período de estudio no era habitual el empadronamiento de la población inmigrante.

Se realizó un análisis descriptivo de las variables anteriores, calculándose los porcentajes de solicitudes de IVE para cada una de ellas. También se calcularon las tasas anuales por 1.000 mujeres de 15 a 49 años y tasas medias por gestación menor, mayor o igual de 12 semanas por tipo de población; no se han calculado tasas por grupos de edad al no disponer del número de mujeres inmigrantes estratificados por edad. Se calcularon RR con sus IC al $95 \%$ para estudiar la relación entre población inmigrante y no inmigrante, y el número de IVE por años, utilizando el test de chi cuadrado para calcular la significación estadística aplicando el programa Epi Info. Para conocer la situación de inmigración en la solicitud se tuvo en cuenta el número de la tarjeta de reconocimiento temporal (todas empiezan por 60/) y/o el número de pasaporte.

\section{RESULTADOS}

Se registraron 224 solicitudes de IVE en el período estudiado, el mayor número de solicitudes correspondió al año 2002 (39,7\%), lo que supuso una tasa de IVE de 3,1 (tabla 1). El grupo de edad con mayor número de solicitudes fue el de 20-24 años con 66 (29,5\%) (tabla $1)$, si se tiene en cuenta la situación de inmigrante, también el mayor número correspondió a este grupo de edad, con 40 solicitudes (60,6\%). Sin embargo, para las adolescentes (15-19 años) y para las mujeres entre 35 y 49 años el porcentaje es mayor entre las mujeres no inmigrantes (figura 1 ).

El 95\% de las IVE solicitadas en el período de estudio presentaron menos de 12 semanas de gestación (tabla 1) y este porcentaje se mantuvo al estratificar por inmigrantes y no inmigrantes (figura 2). El 52,7\% de las solicitudes correspondió a mujeres inmigrantes (tabla 1); estratificando por años el porcentaje para esta población ha ido aumentando, oscilando los resultados entre 0\% para el año 1998 y 69,7\% para 2002 (figura 3).

Así mismo, la tasa por 1.000 mujeres de 15 a 49 años fue de 150 en el año 1999 para esta población, seguida del año 2002 con

\section{Figura 1}

\section{Número de solicitudes de IVE según edad y tipo de población, 1998-2002}

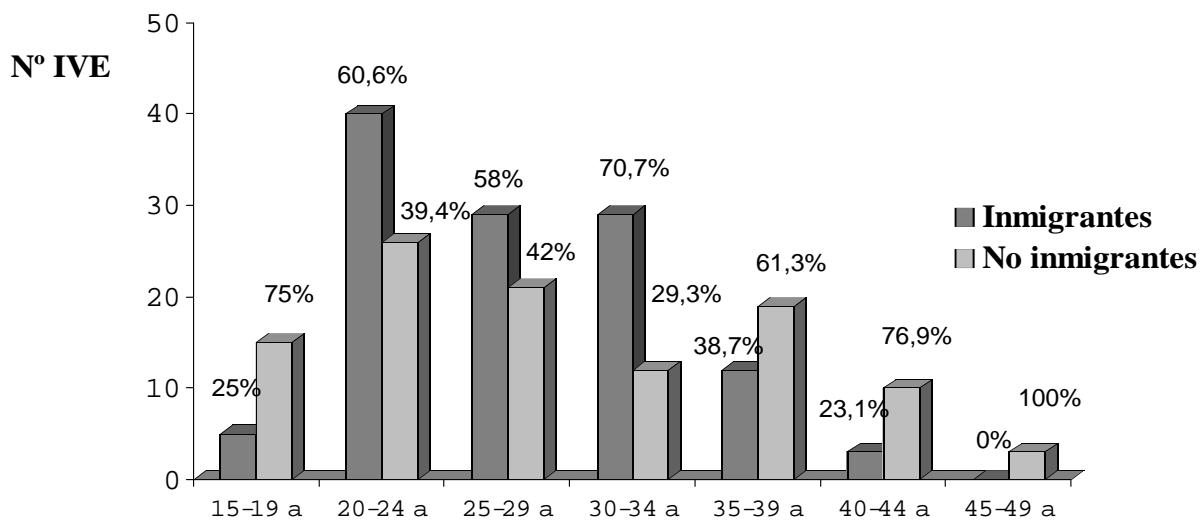


Figura 2

Número de solicitudes de IVE según tipo de población y gestación $\geq o ́$ < de 12 semanas. Período 1998-2002

\section{Mujeres inmigrantes}

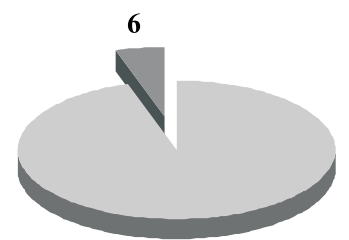

112

$\square 12$ semanas $\square>$ ó $=12$ semanas
Mujeres no inmigrantes

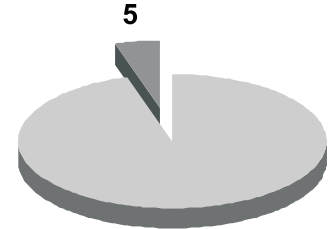

99

- $<12$ semanas $\quad>$ ó $=12$ semanas

Figura 3

Número de solicitudes de IVE según tipo de población. Período 1998-2002

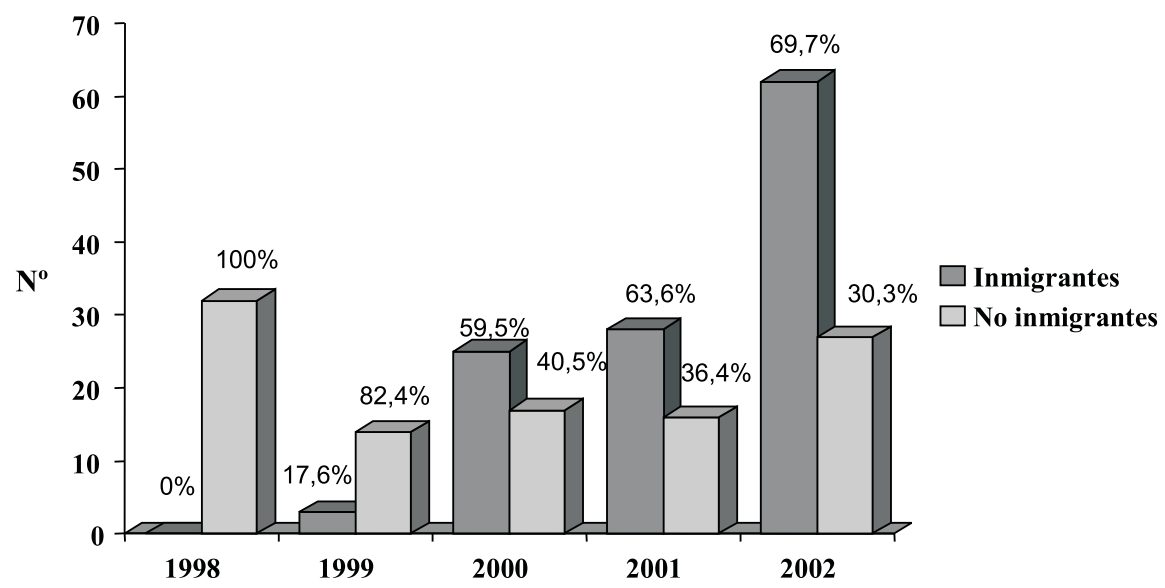

81,6 (figura 4). Con diferencias estadísticamente significativas entre riesgo de solicitar IVE en población inmigrante respecto a la autóctona, oscilando el RR de 66,45 (IC95\%: 36,11-122,28) en el año 2001, a 292,8 (IC95\%: 91,12-940,9) en el 1999 (tabla 2).

\section{DISCUSIÓN}

En el período estudiado se ha encontrado un aumento paulatino de solicitudes de IVE, con mayor número en el año 2002; esto se ha reflejado en un aumento de las tasas en el mismo período. Sin embargo, éstas son menores a las nacionales y a las de Andalucía y aún más bajas que las registradas por otras Comunidades, como Madrid y Cataluña ${ }^{9,16,17}$. Esta diferencia de tasas podría estar de alguna forma influida por dos aspectos: por un lado porque de los tres supuestos que contempla la legislación no se han cuantificado los abortos debidos a malformaciones, ya que éstos se gestionan y realizan en el hospital de Huércal-Overa y no se transmite 
Figura 4

Tasas de IVE por 1000 mujeres de 15 a 49 años, según población. Período 1998-2002

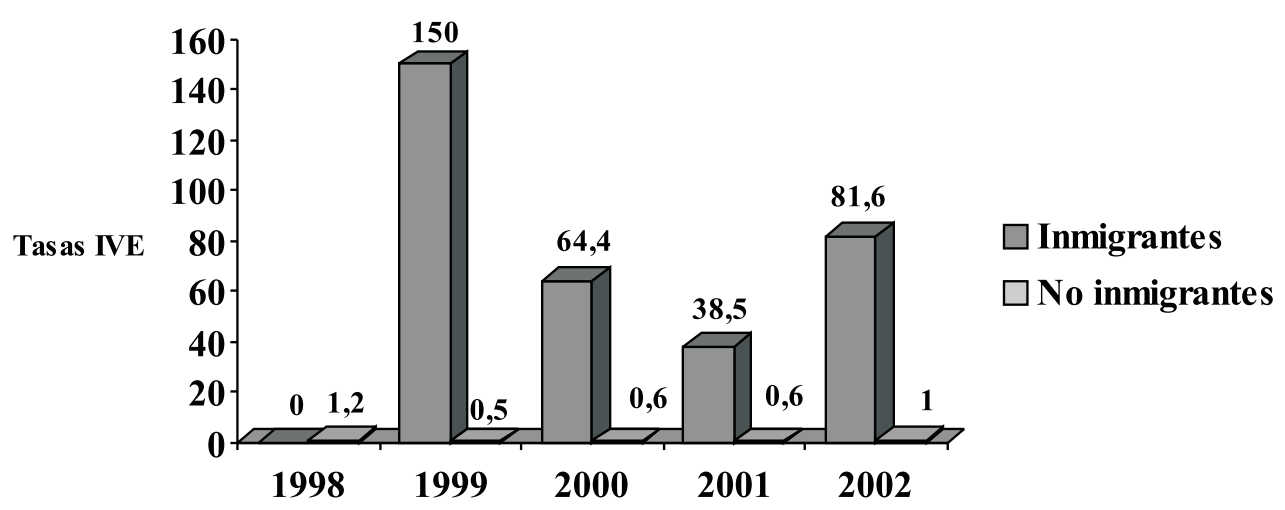

Cálculo de tasas por 1000 mujeres de 15 a 49 años. En población no inmigrante se han utilizado datos de Proyección de población por ámbitos subregionales (IEA): 27164 en 1998, 27338 en 1999, 27451 en 2000, 27606 en 2001 y 27697 en 2002; y en las mujeres inmigrantes Tarjetas de Reconocimiento Temporal: 0 en 1998, 20 en 1999,388 en 2000,728 en 2001 y 760 en 2002.

Tabla 2

Riesgo relativo de solicitar IVE de la población inmigrante respecto a la autóctona

\begin{tabular}{|c|c|c|c|}
\hline Año & RR & IC $_{95 \%}$ & p \\
\hline $1998^{*}$ & --- & --- & -- \\
\hline 1999 & 292,8 & $91,12-940,9$ & $<0,001$ \\
\hline 2000 & 104,04 & $56,65-191,09$ & $<0,001$ \\
\hline 2001 & 66,45 & $36,11-122,28$ & $<0,001$ \\
\hline 2002 & 83,68 & $53,57-130,74$ & $<0,001$ \\
\hline
\end{tabular}

* Al no registrarse ningún caso de aborto en población inmigrante no se ha podido calcular el RR. RR: Riesgo Relativo; IC: Intervalo de Confianza; p: significación estadística.

esta información al distrito; además es difícil estimar su número porque en dicho hospital se realizan las IVE por malformaciones no sólo de este área sino de toda la provincia de Almería. El segundo aspecto que explicaría estas bajas tasas sería que la zona analizada es rural, hábitat en el que en general se realizan menos IVE que en las áreas urbanas y es posible que algunas mujeres, sobre todo autóctonas, acudan a clínicas privadas sin pasar por el médico de familia.
Destaca, no obstante, la tendencia al aumento de las tasas a lo largo del período de estudio, similar a la que se está registrando en Andalucía, Cataluña, Madrid, Murcia y a la media global en España ${ }^{9,16,17}$, aunque distinta a la que siguen otros países como algunos de los nórdicos, en los que se ha registrado una disminución general del aborto ${ }^{6}$.

Se ha detectado un mayor número de solicitudes de IVE en mujeres inmigrantes, con 
tasas superiores a las de mujeres autóctonas. En nuestra zona se ha producido un aumento de esta población desde el año 1999 y algunos autores han indicado que la situación de inmigrante es factor de riesgo para sufrir abortos inducidos ${ }^{10-12}$. Este hecho podría explicarse por las dificultades que conlleva ser inmigrante y por la situación en los países de procedencia, en los que se ha descrito un aumento de embarazos no deseados, entre otros motivos a causa de la falta de conocimiento y disponibilidad de métodos anticonceptivos, desigualdades sociales y demográficas en la salud de las mujeres, etcétera ${ }^{2-5}$. Estos datos no se han podido comparar con los nacionales ni con los de las Comunidades Autónomas, ya que la variable inmigración no se recoge en el documento de solicitud de IVE ni en el cuestionario que posteriormente cumplimentan las clínicas concertadas. Los aspectos comentados anteriormente junto con la forma de cuantificar IVE en mujeres inmigrantes, así como el método utilizado para estimar el tamaño de dicha población, podría conllevar algún sesgo y limitar los resultados del estudio, pero esta valoración puede acercarnos a la realidad de una situación que hasta ahora no está cuantificada y que permitiría realizar los abordajes oportunos. Así mismo, dada la fuente de información utilizada en este trabajo, no se han podido conocer aspectos socioculturales, económicos o sanitarios que pueden influir en la solicitud de IVE, lo que abriría otras posibilidades para futuros estudios.

En la mayoría de los casos la IVE se ha solicitado con menos de 12 semanas de gestación, datos similares al resto de Comunidades Autónomas y a la media nacional que oscilan alrededor de un $90 \%$ de mujeres $^{9,16,17}$. El mayor número de casos se ha producido en el grupo de edad de 20 a 24 años en ambos tipos de población, coincidiendo también con otros registros oficiales ${ }^{9,16,17}$. En el grupo de 15 a 19 años, hay menor número de solicitudes y de éstas la mayoría correspondieron a población autóctona, lo que podría estar influido por el hecho de que en la mayoría de los países el aborto de adolescentes está limitado por restricciones de tipo legal y en muchos, entre ellos España, se requiere el consentimiento de los padres. En Islandia ${ }^{6}$ y en algunos países de Latinoamérica y Caribe son frecuentes los abortos en las adolescentes ${ }^{4}$. No se han podido calcular las tasas por grupos de edad por no disponer de este dato para mujeres inmigrantes.

Con respecto a la incidencia de aborto la situación en Europa es muy variable, influyendo factores culturales, económicos, religiosos y legales. Algunos autores indican que las diferencias pueden ser debidas a distinta disponibilidad y uso de anticoncepti$\operatorname{vos}^{3}$ y que el aumento de su utilización resulta en una menor incidencia del aborto en lugares con fecundidad constante ${ }^{18}$. En la actualidad el aborto quirúrgico es el que se oferta de forma rutinaria ${ }^{19}$. En España, a raíz de la despenalización parcial en el año 1985, se redujeron las desigualdades que implicaba carecer de acceso a esta prestación ${ }^{20}$. En nuestro distrito hasta el año 2001 las IVE se realizaban en dos clínicas concertadas, una en Málaga y otra en Sevilla. El cambio de concierto a la clínica de Almería supuso una disminución de las desigualdades debidas a barreras geográficas, muy importante en nuestra zona, ya que se trata de un distrito sanitario rural del norte de la provincia, con grandes dificultades en los medios de transporte, sobre todo para las mujeres inmigrantes que en muchos casos tenían que desplazarse en autobuses que quedaban lejos del municipio donde vivían.

Hay distintos medios que pueden evitar los embarazos no deseados. En la actualidad los anticonceptivos de emergencia suponen un importante avance para ello y su uso está extendido en muchos países ${ }^{8,21-24}$. En la Comunidad Autónoma Andaluza se ofertó su uso a partir del año 2001, sobre todo para adolescentes. Sería conveniente en próximos estudios, relacionar la utilización de dicho método con una posible disminución del aborto. 
A raíz de estos resultados se iniciaron actuaciones con los profesionales de la zona. En primer lugar, se presentaron los datos a los directivos de las Zonas Básicas de Salud en una Comisión de Dirección en la que se planteó la necesidad de readaptar el programa de planificación familiar a las nuevas necesidades de la población; se discutió la posibilidad de preguntar a la mujer inmigrante en su primera visita al centro sanitario sobre uso de anticonceptivos. Posteriormente se dio a conocer esta información a todos los profesionales que participan en la Red de Vigilancia Epidemiológica, a través del boletín epidemiológico que se elabora entre distrito y hospital, con el fin de que los profesionales pudieran utilizar esta información en la orientación a la mujer, evitando circunstancias y riesgos no deseados y en la medida de lo posible la necesidad de recurrir a la IVE.

\section{BIBLIOGRAFÍA}

1. Hwang AC, Stewart FH. Family planning in the balance. Am J Public Health 2004; 94: 15-18.

2. Goicolea I. Exploring women's needs in an Amazon region of Ecuador. Reprod Health Matters 2001; 9: 193-202.

3. Pinter B. Medico-legal aspects of abortion in Europe. Eur J Contracept Reprod Health Care 2002; 7: 15-19.

4. Langer A. Unwanted pregnancy: impact on health and society in Latin America and the Caribbean. Rev Panam Salud Publica 2002; 11: 192-204.

5. Kalediene R, Nadisauskiene R. Women's health, changes and challenges in health policy development in Lithuania. Reprod Health Matters 2002; 10: 117-126.

6. Knudsen LB, Gissler M, Bender SS, Hedberg C, Ollendorff U, Sundstrom K et al. Induced abortion in the Nordic countries: special emphasis on young women. Acta Obstet Gynecol Scand 2003; 82: 257 268.

7. Xinh TT, Binh PT, Phuong VH, Goto A. Counseling about contraception among repeated aborters in Ho Chi Minh City, Vietnam. Health Care Women Int 2004; 25: 20-39.
8. Heimburger A, Gras C, Guedes A. Expanding access to emergency contraception: the case of Brazil and Colombia. Reprod Health Matters 2003; 11: 150-160.

9. Interrupción Voluntaria del Embarazo. Andalucía, 2001-2002. Evolución de la Incidencia de IVE 2001-2002. Distribución de las IVE en los Distritos Sanitarios 2001 (Datos 2002 Provisionales). Sevi1la: Consejería de Salud. Junta de Andalucía 2004.

10. Lamur HE. Characteristics of Caribbean-born women having abortions in an Amsterdam clinic. Genus 1993; 49: 135-145.

11. Newell A, Sullivan A, Halai R, Boag F. Sexually transmitted diseases, cervical cytology and contraception in inmigrants and refugees from the former Yugoslavia. Venereology 1998; 11: 25-27.

12. Helstrom L, Odlind V, Zatterstrom C, Johansson M, Granath F, Correia N et al. Abortion rate and contraceptive practices in immigrant and native women in Sweden. Scand J Public Health 2003; 31 : 405-410.

13. Orden de 16 de junio de 1986 sobre estadísticas e información epidemiológica de las interrupciones voluntarias del embarazo realizadas conforme a la Ley Orgánica 9/1985, de 5 de julio (BOE de 3 de julio de 1986).

14. Ley Orgánica 9/1985, de 5 de julio, de Reforma del artículo 417 bis del Código Penal (BOE de 12 de julio de 1985).

15. Convenio de colaboración en materia de Salud Pública para el colectivo de inmigrantes, suscrito el 19 de marzo de 1999 entre la Consejería de Salud de la Junta de Andalucía y la Federación Andalucía Acoge, la Asociación Médicos del Mundo, la Cruz Roja Española de Andalucía y la Fundación Progreso y Salud.

16. Interrupción Voluntaria del Embarazo: Datos definitivos correspondientes al año 2001. Madrid: Ministerio de Sanidad y Consumo, 2002.

17. Interrupción Voluntaria del Embarazo: Datos definitivos correspondientes al año 2002. Madrid: Ministerio de Sanidad y Consumo, 2004.

18. Marston C, Cleland J. Relationships between contraception and abortion: a review of the evidence. Int Fam Plan Perspect 2003; 29: 6-13.

19. Murthy A, Creinin MD. Pharmacoeconomics of medical abortion: a review of cost in the United 
States, Europe and Asia. Expert Opin Pharmacother 2003 ; 4: 503-513.

20. Peiro R, Colomer C, Alvarez-Dardet C, Ashton JR. Does the liberalisation of abortion laws increase the number of abortions? The case estudy of Spain. Eur J Public Health 2001; 11: 190-194.

21. Díaz-Olavarrieta C, Turner AN, Ellertson C, Helzner JF, Ezcurra E. Policy climate, scholarship, and provision of emergency contraception at affiliates of the International Planned Parenthood Federation in Latin America and the Caribbean. Contraception 2002; 65: 143-149.

22. Grimes DA, Raymond EG. Emergency contraception. Ann Intern Med 2002; 137: 180-189.

23. Nelson R. Emergency contraception kept as prescription only in USA. The Lancet 2004; 363: 1707.

24. Robert Steinbrook MD. Waiting for Plan B-The FDA and Nonprescription Use of Emergency Contraception. N Engl J Med 2004; 350: 2327-29. 